\title{
Evaluating the Brief Health Literacy Screen
}

\author{
Kenneth A. Wallston, $\mathrm{PhD}^{7}$, Kathryn M. Goggins, MPH${ }^{2}$, and Sunil Kripalani, MD, MSc ${ }^{1,2,3}$ \\ ${ }^{1}$ School of Nursing, Vanderbilt University, Nashville, TN, USA; ${ }^{2}$ Vanderbilt Center for Health Services Research, Vanderbilt University, Nashville, \\ TN, USA; ${ }^{3}$ Department of Medicine, Vanderbilt University, Nashville, TN, USA.
}

J Gen Intern Med 29(1):22

DOI: $10.1007 / \mathrm{s} 11606-013-2654-3$

(c) Society of General Internal Medicine 2013

The Authors Reply-We thank Louis et al. for their letter commending our article regarding the Brief Health Literacy Screen (BHLS) as used in clinical practice. They state that at their institution they did not find the same magnitude of relationship between the BHLS and the short Test of Functional Health Literacy in Adults (S-TOFHLA) that we found at our institution, and hypothesized that this may have been due to differences in the two patient populations. They speculated that their results might have been less promising than ours because "the tools may perform better in populations with high health literacy."

We agree that there are differences in the two patient populations, but we disagree that the reason we obtained the results we did was due to either racial or educational differences between the two institutions. For one thing, the multiple regression analyses presented in our article adjusted for race and education (along with sex and age) and still found a significant relationship between the BHLS and the S-TOFHLA. Secondly, stimulated by their letter, we examined whether race or educational level moderated our finding in our inpatient sample, where $34 \%$ of the patients $(n=163)$ were African American and $12 \%(n=58)$ had a high school education level or less. Neither race nor educational level made a difference in our findings; the relationship between the BHLS and S-TOFHLA is as strong for Blacks in our sample as it is for Whites, and for those with less education as for those with at least some college.

In Nashville, if not in Chicago, the BHLS appears to validly assess health literacy even among subgroups of patients who have characteristics consistent with lower health literacy. Despite that, we endorse Louis et al.'s call "to further evaluate the utility of the BHLS in diverse patient populations," particularly those which might be suspected of having difficulty understanding medical information unrelated to their demographic characteristics.

Corresponding Author: Sunil Kripalani, MD, MSc; School of Nursing, Vanderbilt University, 1215 21st Avenue South, Nashville, TN, 37232, USA (e-mail: sunil.kripalani@vanderbilt.edu).

Published online October 16, 2013 